Louisiana State University

LSU Digital Commons

Faculty Publications

Department of Mathematics

8-1-2008

\title{
The complexity of recognizing linear systems with certain integrality properties
}

\author{
Guoli Ding \\ Louisiana State University \\ Li Feng \\ The University of Hong Kong \\ Wenan Zang \\ The University of Hong Kong
}

Follow this and additional works at: https://digitalcommons.Isu.edu/mathematics_pubs

\section{Recommended Citation}

Ding, G., Feng, L., \& Zang, W. (2008). The complexity of recognizing linear systems with certain integrality properties. Mathematical Programming, 114 (2), 321-334. https://doi.org/10.1007/s10107-007-0103-y

This Article is brought to you for free and open access by the Department of Mathematics at LSU Digital Commons. It has been accepted for inclusion in Faculty Publications by an authorized administrator of LSU Digital Commons. For more information, please contact ir@lsu.edu. 


\title{
The Complexity of Recognizing Linear Systems with Certain Integrality Properties
}

\author{
Guoli Ding $^{a *}$ Li Feng $^{b} \quad$ Wenan Zang $^{b \dagger}$ \\ a Mathematics Department, Louisiana State University, \\ Baton Rouge, Louisiana, USA \\ ${ }^{b}$ Department of Mathematics, The University of Hong Kong, \\ Hong Kong, China
}

\begin{abstract}
Let $A$ be a $0-1$ matrix with precisely two 1's in each column and let $\mathbf{1}$ be the all-one vector. We show that the problems of deciding whether the linear system $A \boldsymbol{x} \geq \mathbf{1}, \boldsymbol{x} \geq \mathbf{0}$

(1) defines an integral polyhedron,

(2) is totally dual integral (TDI), and

(3) is box-totally dual integral (box-TDI)

are all co- $N P$-complete, thereby confirming the conjecture on $N P$-hardness of recognizing TDI systems made by Edmonds and Giles in 1984.
\end{abstract}

Key words: linear system, polyhedron, total dual integrality, NP-hardness.

${ }^{*}$ Supported in part by NSA grant H98230-05-1-0081 and NSF grants DMS-0556091 and ITR-0326387.

† Supported in part by the Research Grants Council of Hong Kong and Seed Funding for Basic Research of HKU. 


\section{Introduction}

Many combinatorial optimization problems can be naturally formulated as integer linear programs. Due to the special feature of such a problem, sometimes the corresponding linear programming (LP) relaxation yields an optimal solution that is integral, thus solving the problem; sometimes both the LP relaxation and its dual have integral optimal solutions; sometimes box-integrality property holds for the LP relaxation and its dual. While a basic theme in combinatorial optimization is to identify various problems with these properties, the present paper is concerned with hardness of recognizing such scenarios.

A rational linear system $A \boldsymbol{x} \geq \boldsymbol{b}, \boldsymbol{x} \geq \mathbf{0}$ is called totally dual integral (TDI) if the maximization problem in the LP-duality equation

$$
\min \left\{\boldsymbol{w}^{T} \boldsymbol{x} \mid A \boldsymbol{x} \geq \boldsymbol{b}, \boldsymbol{x} \geq \mathbf{0}\right\}=\max \left\{\boldsymbol{y}^{T} \boldsymbol{b} \mid \boldsymbol{y}^{T} A \leq \boldsymbol{w}^{T}, \boldsymbol{y} \geq \mathbf{0}\right\}
$$

has an integral optimal solution $\boldsymbol{y}$ for every integral vector $\boldsymbol{w}$ for which the maximum is finite. Furthermore, system $A \boldsymbol{x} \geq \boldsymbol{b}, \boldsymbol{x} \geq \mathbf{0}$ is called box-totally dual integral (box-TDI) if the system $A \boldsymbol{x} \geq \boldsymbol{b}, \boldsymbol{x} \geq \mathbf{0}, \boldsymbol{u} \geq \boldsymbol{x} \geq \boldsymbol{l}$ is TDI for all rational vectors $\boldsymbol{u}$ and $\boldsymbol{l}$, where coordinates of $\boldsymbol{u}$ are allowed to be $+\infty$. The model of TDI systems plays a crucial role in polyhedral combinatorics, and serves as a general framework for establishing many important results, in particular, min-max relations since, as shown by Edmonds and Giles [8], total dual integrality implies primal integrality: if $A \boldsymbol{x} \geq \boldsymbol{b}, \boldsymbol{x} \geq \mathbf{0}$ is a TDI system and $\boldsymbol{b}$ is integral, then the polyhedron $\{\boldsymbol{x} \mid A \boldsymbol{x} \geq \boldsymbol{b}, \boldsymbol{x} \geq \mathbf{0}\}$ is integral. (As usual, a polyhedron $P$ is called integral if each face of $P$ contains integral vectors.)

A number of well-known results and difficult conjectures in combinatorial optimization can be rephrased by saying that certain polyhedra are integral or certain linear systems are TDI, for instance, the celebrated strong perfect graph theorem obtained recently by Chudnovsky, Robertson, Seymour, and Thomas [4]. So the following recognition problems, all proposed in Schrijver [13], are of both great theoretical interest and practical value.

Problem 1.1 Given a rational linear system, does it determine an integral polyhedron?

Problem 1.2 Given a rational linear system, is it TDI?

Problem 1.3 Given a rational linear system, is it box-TDI?

In connection with Problem 1.2, Edmonds and Giles [9] made the following conjecture.

Conjecture 1.4 It is co-NP-complete to decide whether a given rational linear system is TDI.

The main purpose of this paper is to establish the following result.

Theorem 1.5 Let $A$ be a $0-1$ matrix with precisely two 1's in each column and let 1 be the all-one vector. Then the problems of deciding whether the linear system $A \boldsymbol{x} \geq \mathbf{1}, \boldsymbol{x} \geq \mathbf{0}$

(1) defines an integral polyhedron,

(2) is totally dual integral (TDI), and

(3) is box-totally dual integral (box-TDI)

are all co-NP-complete. 
From this theorem we deduce that Problems 1.1-1.3 are all $N P$-hard. Hence the $N P$-hardness addressed in Conjecture 1.4 is confirmed. In Schrijver [13], it was shown that Problem 1.1 is in co- $N P$, and so are Problems 1.2 and 1.3 if the matrix involved in the linear system is integral. In general, however, the question whether Problems 1.2 and 1.3 are in NP or co- NP is still unanswered.

It is worthwhile pointing out that, first, Problems 1.1-1.3 can all be solved in polynomial time if the rank of the matrix involved in the linear system is a fixed constant, as shown by Cook, Lovász, and Schrijver [6]; second, classical results of Chvátal [5], Fulkerson [10], and Lovász [12] imply that for any $0-1$ matrix $A$, the system $A \mathbf{x} \leq \mathbf{1}, \mathbf{x} \geq \mathbf{0}$ defines an integral polyhedron if and only if it is TDI if and only if $A$ is the clique-vertex incidence matrix of a perfect graph. Since clique-vertex incidence matrices of graphs can be recognized in polynomial time, according to a result of Gilmore on conformal hypergraphs (see page 396 of Berge [1]), the recent breakthrough on perfect graphs $[3,4]$ implies that such a TDI system can be recognized in polynomial time.

The remainder of this paper is organized as follows. In section 2, we show that the problems addressed in Theorem 1.5 are essentially equivalent to the problem of recognizing the so-called quasi-bipartite graphs. In section 3, we verify that it is co-NP-complete to recognize quasi-bipartite graphs, thus proving the theorem. In section 4, we extend two classical min-max theorems on bipartite graphs proved by König and Gupta, respectively, to general graphs, and demonstrate that such a min-max relation holds on a graph $G$ if and only if $G$ is essentially a quasi-bipartite graph.

\section{Equivalence}

To establish the desired complexity results, we shall appeal to a graph recognition problem. A graph $G$ is called quasi-bipartite if for any odd cycle $C$ in $G$, the deletion of all vertices on $C$ from $G$ results in at least one isolated vertex (in other words, $G-V(C)$ contains at least one component which has only one vertex). Note that any bipartite graph is quasi-bipartite. We shall prove that the three problems in our consideration are essentially equivalent to the problem of recognizing quasi-bipartite graphs.

The following theorem gives a structural description of quasi-bipartite graphs.

Theorem 2.1 Let $G=(V, E)$ be a connected simple graph. Then $G$ is quasi-bipartite if and only if either $G$ is $K_{4}$ (the complete graph with four vertices) or there is a partition $\left(X_{1}, X_{2}, Y, Z_{1}, Z_{2}, \ldots, Z_{t}\right)$ (possibly $X_{1} \cup X_{2}=\emptyset$ and $\left.t=0\right)$ of $V$ such that

(i) for each $x \in X_{1}$, the degree $d(x)=1$ and the only neighbor of $x$ is in $X_{2}$;

(ii) each vertex in $X_{2}$ is adjacent to at least one vertex in $X_{1}$, and there is no edge between $X_{2}$ and $Z_{1} \cup Z_{2} \cup \ldots \cup Z_{t}$

(iii) there are $t$ distinct unordered pairs $\left\{y_{1}^{1}, y_{1}^{2}\right\},\left\{y_{2}^{1}, y_{2}^{2}\right\}, \ldots,\left\{y_{t}^{1}, y_{t}^{2}\right\}$ of vertices in $Y$ such that

(a) $y_{i}^{1} \neq y_{i}^{2}$ for $i=1,2, \ldots, t$;

(b) both $y_{i}^{1}$ and $y_{i}^{2}$ are adjacent to all vertices in $Z_{i}$ for $i=1,2, \ldots, t$;

(c) each odd cycle of $G-\left(X_{1} \cup X_{2}\right)$ contains both $y_{i}^{1}$ and $y_{i}^{2}$ for at least one $i$ with $1 \leq i \leq t$;

(iv) $\left|Z_{i}\right| \geq 2$ for $i=1,2, \ldots, t$, and the degree $d(z)=2$ for all $z \in Z_{1} \cup Z_{2} \cup \ldots \cup Z_{t}$. 
Proof. The "if" part follows instantly from the conditions (i)-(iv) listed above. So we proceed to the "only if" part and assume $G \neq K_{4}$. We need to identify a required partition of $V$.

The choices for $X_{1}$ and $X_{2}$ are clear. Let $X_{1}$ denote the set of all vertices $x$ of $G$ with $d(x)=1$, and let $X_{2}$ denote the set of all vertices in $V-X_{1}$ which are adjacent to at least one vertex of $X_{1}$ in $G$.

(1) For each odd cycle $D$ in $G-\left(X_{1} \cup X_{2}\right)$, there exists a vertex $w$ in $G-\left(X_{1} \cup X_{2} \cup V(D)\right)$ such that the neighbors of $w$ in $G$ are all contained in $D$.

Since $G$ is quasi-bipartite and $D$ is an odd cycle, by definition there exists a vertex $w \in G-V(D)$ such that the neighbors of $w$ in $G$ are all contained in $D$. Note that $w \notin X_{1}$ (for otherwise $D$ would contain vertices in $X_{2}$ ) and that $w \notin X_{2}$ (for otherwise $w$ has a neighbor in $X_{1}$, which is outside $D$ ). Thus (1) holds.

The main part of this proof is to show the following, which will give us the desired partition.

(2) For each odd cycle $C$ in $G-\left(X_{1} \cup X_{2}\right)$, there exist a pair of distinct vertices $\{u, v\}$ on $C$ and a subset $W$ of $V-\left(X_{1} \cup X_{2} \cup\{u, v\}\right)$ (possibly $W \cap V(C) \neq \emptyset$ ) such that

- $|W| \geq 2$; and

- each vertex $w \in W$ has degree two in $G$ and is adjacent to both $u$ and $v$.

We prove (2) by contradiction. Suppose some odd cycle $C$ in $G-\left(X_{1} \cup X_{2}\right)$ is a counterexample to (2) with the shortest length.

(3) We may assume that $C$ is an induced cycle in $G$, for otherwise let $C^{\prime}$ be an odd cycle formed by a chord and a segment of $C$. Then $\left|V\left(C^{\prime}\right)\right|<|V(C)|$. By the minimality of $C$, there exist a pair of distinct vertices $\{u, v\}$ on $C^{\prime}$ and a subset $W$ of $V-\left(X_{1} \cup X_{2} \cup\{u, v\}\right)$ with the properties as described in (2). Thus (2) holds for $C$ with respect to $\{u, v\}$ and $W$, a contradiction.

(4) $C$ is not a triangle (a cycle of length three).

Suppose the contrary: $C$ is a triangle $a b c$. We claim that

(5) There exists a vertex $w \notin X_{1} \cup X_{2} \cup\{a, b, c\}$ which has degree two in $G$ and is adjacent to precisely two of $a, b, c$.

To justify (5), let $s$ be a vertex in $G-\left(X_{1} \cup X_{2} \cup V(C)\right)$ such that the neighbors of $s$ in $G$ are all contained in $C$ (recall (1)). Since $s \notin X_{1}$ and $|V(C)|=3$, we have $d(s)=2$ or 3 . If $d(s)=2$ then $w=s$ is as desired. So we assume $d(s)=3$. For any pair of vertices $\{p, q\}$ on $C$, by $(1)$, there exist a vertex $t_{p q}$ in $G-\left(X_{1} \cup X_{2} \cup\{p, q, s\}\right)$ such that the neighbors of $t_{p q}$ in $G$ are all contained in $\{p, q, s\}$. Observe that for at least one such pair $\{p, q\}$, vertex $t_{p q}$ is not the one in $\{a, b, c\}-\{p, q\}$, for otherwise $s, a, b, c$ all have degree three in $G$ and hence $G=K_{4}$, a contradiction. Since the neighbors of $s$ are $a, b, c$, this $t_{p q}$ is not adjacent to $s$. From $t_{p q} \notin X_{1} \cup X_{2} \cup\{a, b, c\}$, we deduce that $w=t_{p q}$ is as desired. Thus claim (5) is established.

Let $w$ be the vertex as specified in (5). Rename the vertices if necessary, we may assume that $a, b$ are the only neighbors of $w$. Then (1) guarantees the existence of a vertex $w^{\prime}$ in $G-\left(X_{1} \cup X_{2} \cup\{a, b, w\}\right)$ which is adjacent to no vertex outside $\{a, b, w\}$ in $G$. Since $w^{\prime} \notin X_{1}$, we see that $w^{\prime}$ has degree two in $G$ and is adjacent to both $a$ and $b$. Set $W=\left\{w, w^{\prime}\right\}$. Then (2) holds with respect to vertex pair $\{a, b\}$ and $W$, this contradiction yields (4). 
Let $c_{0}, c_{1}, \ldots, c_{2 k}$ be all the vertices on $C$ in cyclic order. By (4), we have

(6) $k \geq 2$.

(7) Let $p$ be an arbitrary vertex outside $C$. If $p$ is adjacent to no vertex outside $C$, then $p$ has degree two in $G$ and is adjacent to $c_{i}$ and $c_{i+2}$ for some $i$ with $0 \leq i \leq k$, where the subscripts are taken modulo $2 k+1$.

To justify (7), note first that $p \notin X_{1} \cup X_{2}$ for $C$ contains no vertex in $X_{1} \cup X_{2}$ (recall the definitions of $X_{1}$ and $X_{2}$ ). So $d(p) \geq 2$. If $d(p)=2$ but the neighbors of $p$ are not of distance two on $C$ or if $d(p) \geq 3$ then, by (6), we can find an induced odd cycle $C^{\prime}$ formed by $p$ and a segment of $C$ such that $\left|V\left(C^{\prime}\right)\right|<|V(C)|$. Thus the minimality of $C$ guarantees the existence of a vertex pair $\{u, v\}$ on $C^{\prime}$ and a subset $W$ of $V-\left(X_{1} \cup X_{2} \cup\{u, v\}\right)$ with properties as described in (2). Since all neighbors of $p$ are contained in $C$, they must have degree at least three and hence must be all outside $W$. So $p \notin\{u, v\}$. It follows that (2) also holds for $C$ with respect to $\{u, v\}$ and $W$, this contradiction establishes (7).

Suppose $p$ is a vertex outside $C$ such that $p$ has degree two in $G$ and is adjacent to both $c_{i}$ and $c_{i+2}$ for some $i$ with $0 \leq i \leq k$, where the subscripts are taken modulo $2 k+1$. Notice that there is no second vertex $q(\neq p)$ outside $C$ such that $q$ has degree two in $G$ and also has $c_{i}$ and $c_{i+2}$ as its neighbors, since otherwise the vertex pair $\left\{c_{i}, c_{i+2}\right\}$ and set $W=\{p, q\}$ would satisfy (2) with respect to $C$, a contradiction. For convenience, let $p_{i+1}$ denote the unique vertex outside $C$ (if any) such that $p_{i+1}$ has degree two in $G$ and is adjacent to both $c_{i}$ and $c_{i+2}$, and let $P$ denote the set of all these $p_{i+1}$ 's. Since $G$ is quasi-bipartite, by (7) we have $P \neq \emptyset$. Rename the vertices of $C$ if necessary, we may assume that $p_{0}$ exists. Let $i_{0}, i_{1}, \ldots, i_{l}$ be the longest subsequence of the sequence $0,1, \ldots, 2 k$ with the following properties:

(a) $i_{0}<i_{1}<\ldots<i_{l}, i_{0}=0$, and $i_{l} \leq 2 k-1$;

(b) for each $j$ with $j \geq 1$, term $i_{j}$ is the smallest subscript $t$ such that $t \geq i_{j-1}+2$ and that $p_{t} \in P$. Observe that

(8) $d\left(c_{i_{j}}\right) \geq 3$ for each $j=0,1, \ldots, l$, since otherwise the vertex pair $\left\{c_{i_{j}-1}, c_{i_{j}+1}\right\}$ and set $W=\left\{p_{i_{j}}, c_{i_{j}}\right\}$ would satisfy (2) with respect to $C$, a contradiction.

Let $C^{\prime}$ be the odd cycle obtained from $C$ by replacing all $c_{i_{j}}$ with $p_{i_{j}}$ for $0 \leq j \leq l$. We propose to show that

(9) There is no vertex outside $C^{\prime}$ whose neighbors are all contained in $C^{\prime}$.

To justify (9), we assume the contrary: some vertex $q$ outside $C^{\prime}$ has all neighbors in $C^{\prime}$. Since $d\left(p_{i_{j}}\right)=2$, vertex $q$ is not adjacent to $p_{i_{j}}$ for any $j=0,1, \ldots, l$. So $q$ has all neighbors in $C$. In view of (3) and (8), vertex $q \neq c_{i_{j}}$ for any $j=0,1, \ldots, l$, and so $q$ is outside $C$. It follows from (7) that $q$ is some $p_{t}$ in $P$. According to selection (b), we must either have some $j$ with $0 \leq j \leq l$ such that $t=i_{j}+1$ or have $t=2 k$. Thus $q$ has at least one neighbor $c_{i_{j}}$ or $c_{0}$ outside $C^{\prime}$, contradicting the choice of $q$. Thus (9) follows.

From (9) we conclude that $G$ is not a quasi-bipartite graph, this contradiction completes the proof of (2). Let us call a pair $\{u, v\}$ of vertices a blocking pair if there exist an odd cycle $C$ in $G-\left(X_{1} \cup X_{2}\right)$ and a set $W$ of vertices that have the properties as described in (2).

(10) If $\{u, v\}$ is a blocking pair then $d(u) \geq 3$ and $d(v) \geq 3$. 
If (10) was false then at least one of $u$ and $v$, say $u$, has degree at most two, which implies $d(u)=2$ since $u \notin X_{1}$. By the definition of a blocking pair, the corresponding set $W$ must contain precisely two vertices, say $w_{1}$ and $w_{2}$. Since $d\left(w_{1}\right)=d\left(w_{2}\right)=2$, it follows that the only cycle containing $u$ is the one induced by $\left\{u, v, w_{1}, w_{2}\right\}$, contradicting the assumption that $\{u, v\}$, as a blocking pair, is contained in an odd cycle. This contradiction proves (10).

Let $\left\{u_{1}, v_{1}\right\},\left\{u_{2}, v_{2}\right\}, \ldots,\left\{u_{t}, v_{t}\right\}$ be all the blocking pairs and let $W_{1}, W_{2}, \ldots, W_{t}$ be the corresponding sets of vertices. For any two distinct indices $i, j \in\{1,2, \ldots, t\}$, we have $W_{i} \cap W_{j}=\emptyset$ since $\left\{u_{i}, v_{i}\right\} \neq\left\{u_{j}, v_{j}\right\}$. Moreover, $W_{i} \cap\left\{u_{j}, v_{j}\right\}=\emptyset$ since vertices in $W_{i}$ have degree two while, by (10), $u_{j}$ and $v_{j}$ each has degree at least three. Therefore, setting $\left\{y_{i}^{1}, y_{i}^{2}\right\}=\left\{u_{i}, v_{i}\right\}$ and $Z_{i}=W_{i}$ for $i=1,2, \ldots, t$, and then $Y=V(G)-\left(X_{1} \cup X_{2} \cup Z_{1} \cup Z_{2} \cup \ldots \cup Z_{t}\right)$ defines a required partition.

Let $A$ be a $0-1$ matrix with precisely two 1's in each column and with dimension $m \times n$. To matrix $A$, we associate a graph $G[A]=(V, E)$ with $m$ vertices and $n$ edges as follows: each vertex $i$ corresponds to row $i$ of $A$, and each edge $i j$ corresponds to a column of $A$ in which the two 1 's are contained in row $i$ and row $j$. (Possibly $G[A]$ contains parallel edges.) From the definition we see that $A$ is nothing but the vertex-edge incidence graph of $G[A]$.

Lemma 2.2 Let $A$ be a 0-1 matrix with precisely two 1's in each column, let 1 be the all-one vector, and let $G[A]=(V, E)$ be the graph associated with $A$. If the system $A \boldsymbol{x} \geq \mathbf{1}, \boldsymbol{x} \geq \mathbf{0}$ defines an integral polyhedron, then $G[A]$ is quasi-bipartite.

Proof. Assume the contrary: for some odd cycle $C$ of $G[A]$, no component of $G[A]-V(C)$ contains only one vertex. Let $\boldsymbol{w}$ be the following weight function defined on $E: w_{e}=0$ for all edges $e$ in $G[A]-V(C)$ and 1 otherwise. Next, let $\boldsymbol{x}^{*}$ be the vector defined on $E$ such that $x_{e}^{*}=1 / 2$ if $e$ is an edge on $C, x_{e}^{*}=1$ if $e$ is an edge in $G[A]-V(C)$, and $x_{e}^{*}=0$ otherwise. Finally, let $\boldsymbol{y}^{*}$ be the vector defined on $V$ such that $y_{v}^{*}=1 / 2$ if $v$ is a vertex on $C$ and 0 otherwise. Since each component of $G[A]-V(C)$ contains at least one edge, $\boldsymbol{x}^{*}$ and $\boldsymbol{y}^{*}$ are feasible solutions to the primal and dual in the following LP-duality equation, respectively.

$$
\min \left\{\boldsymbol{w}^{T} \boldsymbol{x} \mid A \boldsymbol{x} \geq \mathbf{1}, \boldsymbol{x} \geq \mathbf{0}\right\}=\max \left\{\boldsymbol{y}^{T} \mathbf{1} \mid \boldsymbol{y}^{T} A \leq \boldsymbol{w}^{T}, \boldsymbol{y} \geq \mathbf{0}\right\}
$$

Note that $\boldsymbol{w}^{T} \boldsymbol{x}^{*}=\left(\boldsymbol{y}^{*}\right)^{T} \mathbf{1}=(2 k+1) / 2$, where $2 k+1$ is the length of $C$. By the LP-duality theorem, $\boldsymbol{x}^{*}$ and $\boldsymbol{y}^{*}$ are optimal solutions to the primal and dual in (2.1), respectively. Since $\boldsymbol{w}$ is an integral vector while the optimal value of $(2.1)$ is $(2 k+1) / 2$ (not integral), the system $A \boldsymbol{x} \geq \mathbf{1}, \boldsymbol{x} \geq \mathbf{0}$ does not define an integral polyhedron, a contradiction.

The following theorems assert that the problems addressed in Theorem 1.5 are essentially equivalent to the problem of recognizing quasi-bipartite graphs.

Theorem 2.3 Let $A$ be a 0-1 matrix with precisely two 1's in each column, let 1 be the all-one vector, and let $G[A]=(V, E)$ be the graph associated with $A$. Then the following statements are equivalent:

(i) The system $A \boldsymbol{x} \geq \mathbf{1}, \boldsymbol{x} \geq \mathbf{0}$ is TDI;

(ii) The system $A \boldsymbol{x} \geq \mathbf{1}, \boldsymbol{x} \geq \mathbf{0}$ is box-TDI;

(iii) The graph $G[A]$ is quasi-bipartite, and no component of $G[A]$ contains $K_{4}$ as a spanning subgraph. 
Theorem 2.4 Let $A$ be a $0-1$ matrix with precisely two 1's in each column, let 1 be the all-one vector, and let $G[A]=(V, E)$ be the graph associated with $A$. Then the following statements are equivalent:

(i) The system $A \boldsymbol{x} \geq \mathbf{1}, \boldsymbol{x} \geq \mathbf{0}$ defines an integral polyhedron;

(ii) The graph $G[A]$ is quasi-bipartite.

Let us present the proofs of these two theorems.

Proof of Theorem 2.3. It is well known that (ii) $\Rightarrow$ (i).

(i) $\Rightarrow$ (iii). By Lemma 2.2, $G[A]$ is quasi-bipartite. To show the remaining statement, we assume the contrary: some component $\Omega$ of $G[A]$ contains $K_{4}$ as a spanning subgraph. Let $\boldsymbol{w}$ be the following weight function defined on $E: w_{e}=1$ for all edges $e$ in $\Omega$ and 0 otherwise. Then $\min \left\{\boldsymbol{w}^{T} \boldsymbol{x} \mid A \boldsymbol{x} \geq \mathbf{1}, \boldsymbol{x} \geq\right.$ $\mathbf{0}$, integer $\}=2$, while $\max \left\{\boldsymbol{y}^{T} \mathbf{1} \mid \boldsymbol{y}^{T} A \leq \boldsymbol{w}^{T}, \boldsymbol{y} \geq \mathbf{0}\right.$, integer $\}=1$. Thus, by the Edmonds-Giles theorem [8], $A \boldsymbol{x} \geq \mathbf{1}, \boldsymbol{x} \geq \mathbf{0}$ is not TDI, a contradiction.

(iii) $\Rightarrow$ (ii). We aim to show that the system $A \boldsymbol{x} \geq \mathbf{1}, \boldsymbol{u} \geq \boldsymbol{x} \geq \boldsymbol{l}, \boldsymbol{x} \geq \mathbf{0}$ is TDI for all rational vectors $\boldsymbol{u}$ and $\boldsymbol{l}$ defined on $E$. This amounts to showing that for any integral vector $\boldsymbol{w}$ defined on $E$ such that the maximum (and hence the optimal value) of the following LP-duality equation

$$
\min \left\{\boldsymbol{w}^{T} \boldsymbol{x}\left[\begin{array}{c}
A \\
I \\
-I
\end{array}\right] \boldsymbol{x} \geq\left[\begin{array}{c}
\mathbf{1} \\
\boldsymbol{l} \\
-\boldsymbol{u}
\end{array}\right], \boldsymbol{x} \geq \mathbf{0}\right\}=\max \left\{\boldsymbol{y}^{T}\left[\begin{array}{c}
\mathbf{1} \\
\boldsymbol{l} \\
-\boldsymbol{u}
\end{array}\right] \boldsymbol{y}^{T}\left[\begin{array}{c}
A \\
I \\
-I
\end{array}\right] \leq \boldsymbol{w}^{T}, \boldsymbol{y} \geq \mathbf{0}\right\}
$$

is finite, the maximization problem in (2.2) has an integral optimal solution. To this end, let $\boldsymbol{y}^{*}$ be an optimal solution to the maximization problem in (2.2) and let $\eta_{i}^{*}$ denote the $i$ th entry of $\boldsymbol{y}^{*}$. (We shall also use $\eta_{i}$ to denote the $i$ th entry of $\boldsymbol{y}$.)

For convenience, let $G^{\prime}=\left(V, E^{\prime}\right)$ be the simple graph defined as follows: $i j \in E^{\prime}$ if and only if there is at least one edge between $i$ and $j$ in $G$. Clearly $G^{\prime}$ is quasi-bipartite if and only if so is $G$. From hypothesis (iii), it can be seen that no component of $G^{\prime}$ is $K_{4}$. So the vertex set $V$ of $G^{\prime}$ (and hence of $G[A]$ ) admits a partition $\left(X_{1}, X_{2}, Y, Z_{1}, Z_{2}, \ldots, Z_{t}\right)$ (possibly $X_{1} \cup X_{2}=\emptyset$ and $t=0$ ) with properties (i)-(iv) as described in Theorem 2.1 .

(1) We may assume that $\eta_{i}^{*}=0$ for any vertex $i \in X_{2}$.

Otherwise, let $j$ be a vertex in $X_{1}$ such that $i$ is the only neighbor of $j$ in $G$. Define a vector $\overline{\boldsymbol{y}}$ such that $\bar{\eta}_{i}=0, \bar{\eta}_{j}=\eta_{i}^{*}+\eta_{j}^{*}$, and $\bar{\eta}_{k}=\eta_{k}^{*}$ for any $k \neq i, j$. Clearly, $\overline{\boldsymbol{y}}$ is a feasible solution to the maximization problem in (2.2) and has the same objective value as $\boldsymbol{y}^{*}$. So $\overline{\boldsymbol{y}}$ is also an optimal solution to the maximization problem. Repeatedly applying the same procedure, we have (1).

(2) We may assume that for each odd cycle $C$ in $G[A]$, there is a vertex $i$ on $C$ such that $\eta_{i}^{*}=0$.

Otherwise, $C$ contains no vertex in $X_{1} \cup X_{2}$ by the definition of $X_{1}$ and by (1). In view of Theorem 2.1, there exist a vertex pair $\left\{y_{k}^{1}, y_{k}^{2}\right\}$ on $C$ and a vertex subset $Z_{k}$ outside $X_{1} \cup X_{2} \cup\left\{y_{k}^{1}, y_{k}^{2}\right\}$ such that

- each vertex in $Z_{k}$ is adjacent to both $y_{k}^{1}$ and $y_{k}^{2}$ but nonadjacent to any other vertex in $G$;

- there is no edge between any two vertices in $Z_{k}$; and

- $\left|Z_{k}\right| \geq 2$. 
Let $z_{1}, z_{2}$ be two vertices in $Z_{k}$, and let $\eta_{p}^{*}, \eta_{q}^{*}, \eta_{r}^{*}, \eta_{s}^{*}$ be the entries of $\boldsymbol{y}^{*}$ corresponding to vertices $y_{k}^{1}, y_{k}^{2}, z_{1}, z_{2}$, respectively. Set $\delta=\min \left\{\eta_{p}^{*}, \eta_{q}^{*}\right\}$, and define a vector $\overline{\boldsymbol{y}}$ such that $\bar{\eta}_{i}=\eta_{i}^{*}-\delta$ if $i=p$ or $q, \bar{\eta}_{i}=\eta_{i}^{*}+\delta$ if $i=r$ or $s$, and $\bar{\eta}_{i}=\eta_{i}^{*}$ for any $i \neq p, q, r, s$. Clearly, $\overline{\boldsymbol{y}}$ is a feasible solution to the maximization problem in (2.2) and has the same objective value as $\boldsymbol{y}^{*}$. So $\overline{\boldsymbol{y}}$ is also an optimal solution to the maximization problem. Note that at least one of $\bar{\eta}_{p}$ and $\bar{\eta}_{q}$ is zero. Repeatedly applying the same procedure, we get (2).

Let $U$ denote the set of all vertices $i$ with $\eta_{i}^{*}=0$, and let $\bar{A}$ be the matrix obtained from $A$ by deleting the rows corresponding to all vertices $i$ in $U$. Then

(3) $\bar{A}$ is a totally unimodular matrix.

To justify (3), notice that $G-U$ is a bipartite graph by (2). It follows that the vertex-edge incidence matrix $M$ of $G-U$ is totally unimodular (see page 273 of Schrijver [13]). Clearly, $\bar{A}$ is obtained from $M$ by adding $0-1$ columns with at most one 1 . By definition, it is easy to see that $\bar{A}$ is also a totally unimodular matrix, so (3) is proved.

Once again from the definition we deduce that

(4) $\left[\begin{array}{c}\bar{A} \\ I \\ -I\end{array}\right]$ is also a totally unimodular matrix.

Consider the following LP problem

$$
\max \left\{\boldsymbol{z}^{T}\left[\begin{array}{c}
\mathbf{1} \\
\boldsymbol{l} \\
-\boldsymbol{u}
\end{array}\right] \boldsymbol{z}^{T}\left[\begin{array}{c}
\bar{A} \\
I \\
-I
\end{array}\right] \leq \boldsymbol{w}^{T}, \boldsymbol{z} \geq \mathbf{0}\right\} .
$$

Let $\overline{\boldsymbol{z}}$ be the vector obtained from $\boldsymbol{y}^{*}$ by deleting all entries $\eta_{i}^{*}$ with $i \in U$. Clearly $\overline{\boldsymbol{z}}$ is a feasible solution to $(2.3)$. So

(5) The optimal value of $(2.2)=$ the objective value of $(2.2)$ corresponding to $\boldsymbol{y}^{*}=$ the objective value of (2.3) corresponding to $\bar{z} \leq$ the optimal value of (2.3).

In view of (4), (2.3) has an integral optimal solution $\boldsymbol{z}^{*}$ (see Theorem 19.3 (ii) of Schrijver [13]). Let $\overline{\boldsymbol{y}}$ be the vector obtained from $\boldsymbol{z}^{*}$ by adding entries $\bar{\eta}_{i}=0$ for all $i \in U$. Clearly $\overline{\boldsymbol{y}}$ is a feasible solution to (2.2) and is integral. So

(6) The optimal value of $(2.3)=$ the objective value of (2.3) corresponding to $\boldsymbol{z}^{*}=$ the objective value of (2.2) corresponding to $\overline{\boldsymbol{y}} \leq$ the optimal value of $(2.2)$.

Combining (5) and (6), we see that all inequalities in (5) and (6) hold with equalities, and hence $\overline{\boldsymbol{y}}$ is an integral optimal solution to the maximization problem in (2.2), completing the proof.

Proof of Theorem 2.4. The implication (i) $\Rightarrow($ ii) is already established in Lemma 2.2. Let us show that (ii) $\Rightarrow(\mathrm{i})$. For this purpose, we turn to prove that

(1) If $G[A]$ contains $K_{4}$ as a spanning subgraph, then (i) holds.

Let $Q_{6}$ be the vertex-edge incidence matrix of $K_{4}$, which is also the triangle-edge incidence matrix of $K_{4}$ (since the planar dual of $K_{4}$ is also $K_{4}$ ). Then $A$ is obtained from $Q_{6}$ by duplicating some columns. Let $\boldsymbol{w}$ 
be an arbitrary nonnegative integral weight function defined on $E$. We define a vector $\overline{\boldsymbol{w}}$ on the edge set of $K_{4}$, such that $\bar{w}_{i j}$ is the smallest weight of all edges between $i$ and $j$ in $G$ for all $1 \leq i<j \leq 4$. It is a routine matter to check that

(2) The minimization problem in (2.1) has an integral optimal solution if and only if the problem $\min \left\{\overline{\boldsymbol{w}}^{T} \boldsymbol{z} \mid Q_{6} \boldsymbol{z} \geq \mathbf{1}, \boldsymbol{z} \geq \mathbf{0}\right\}$ has an integral optimal solution.

It was shown by Seymour $[7,15]$ that system $Q_{6} \boldsymbol{z} \geq \mathbf{1}, \boldsymbol{z} \geq \mathbf{0}$ defines an integral polyhedron. So $\min \left\{\overline{\boldsymbol{w}}^{T} \boldsymbol{z} \mid Q_{6} \boldsymbol{z} \geq \mathbf{1}, \boldsymbol{z} \geq \mathbf{0}\right\}$ has an integral optimal solution, and hence so does the minimization problem in (2.1) by (2). Therefore (1) is established.

Applying (1) and Theorem 2.3 to each component of $G[A]$, we get the desired statement.

\section{Complexity}

Given the equivalence established in the preceding section, we are ready to prove Theorem 1.5. Let us first consider the aforementioned graph recognition problem, and then we shall reduce the problems addressed in Theorem 1.5 to this one.

\section{Quasi-bipartite recognition problem}

InSTANCE: A connected simple graph $G=(V, E)$ with minimum degree at least three and a set of vertex pairs in $G$.

QuEstion: Does every odd cycle in $G$ contain at least one of these pairs?

Theorem 3.1 The quasi-bipartite recognition problem is co-NP-complete.

Proof. Obviously, the quasi-bipartite recognition problem is in co- $N P$. To prove the assertion, it suffices to reduce the 3 -SATISFIABILITY problem $(3 S A T)[11]$ to the complement of this problem. Let $U=\left\{u_{1}, u_{2}, \ldots, u_{n}\right\}$ be the set of variables and let $\mathcal{C}=\left\{C_{1}, C_{2}, \ldots, C_{m}\right\}$ be the set of clauses in an arbitrary instance of $3 S A T$ in $C N F$. We aim to construct a connected simple graph $G=(V, E)$ with minimum degree at least three and a set of vertex pairs in $G$ so that there exists an odd cycle in $G$ that contains none of these vertex pairs if and only if $\mathcal{C}$ is satisfiable. The construction goes as follows:

(1) For each variable $u_{i} \in U$, there is a truth-setting component $T_{i}$, which is obtained from the cycle $a_{2 i-2} a_{2 i-1}^{1} a_{2 i} a_{2 i-1}^{2} a_{2 i-2}$ by adding one special edge $a_{2 i-1}^{1} a_{2 i-1}^{2}$. Note that $T_{i}$ and $T_{i+1}$ have precisely one vertex $a_{2 i}$ in common.

(2) For each clause $C_{j} \in \mathcal{C}$, there is a satisfaction-testing component $S_{j}$, which is the union of three internally disjoint paths $b_{2 j-2} b_{2 j-1}^{k} b_{2 j}$ for $k=1,2,3$. Note that $S_{j}$ and $S_{j+1}$ have precisely one vertex $b_{2 j}$ in common.

(3) For each clause $C_{j} \in \mathcal{C}$, let $z_{j}^{1}, z_{j}^{2}$, and $z_{j}^{3}$ denote the three literals in $C_{j}$. For each literal $z_{j}^{k}$, if $z_{j}^{k}=u_{p}$ for some $p$, then add one special edge $b_{2 j-1}^{k} a_{2 p-1}^{2}$; if $z_{j}^{k}=\bar{u}_{p}$ for some $p$, then add one special edge $b_{2 j-1}^{k} a_{2 p-1}^{1}$. 
(4) Finally, add one edge $a_{0} b_{2 m}$ and identify $b_{0}$ with $a_{2 n}$.

The construction of $G$ is completed, and the vertex pairs are precisely endvertex pairs of special edges. An illustration is depicted in Figure 1. It is easy to see that the construction can be accomplished in polynomial time and the resulting graph has $3 n+4 m+1$ vertices and $5 n+9 m+1$ edges (in which $n+3 m$ edges are special).

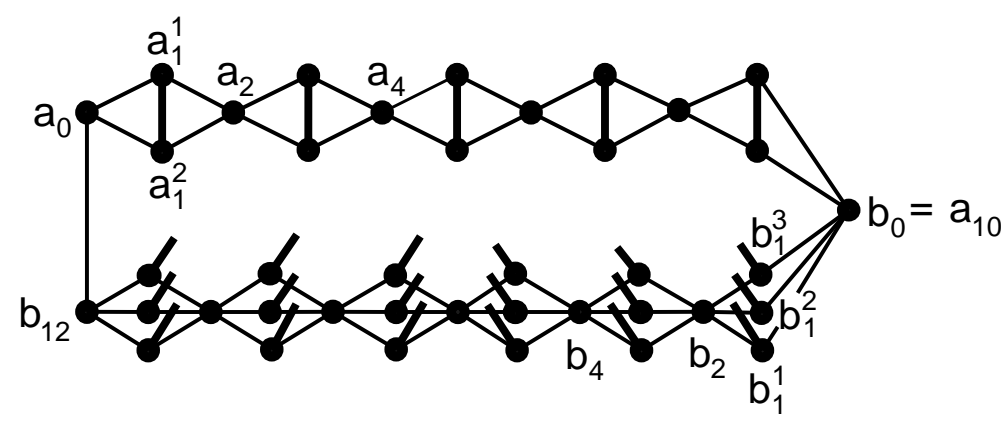

Figure 1. A partially constructed graph, with $n=5$ and $m=6$.

Let us show that there exists an odd cycle in $G$ that contains none of these vertex pairs if and only if $\mathcal{C}$ is satisfiable.

Sufficiency. Suppose that $\tau: U \rightarrow\{$ true, false $\}$ is a satisfying truth assignment for $\mathcal{C}$. Then there exists at least one true literal in each clause. We choose one (denote it by $z_{j}^{h_{j}}$ ) from each clause $C_{j}$ such that $\tau\left(z_{j}^{h_{j}}\right)=$ true. For each variable $u_{i}$, if $\tau\left(u_{i}\right)=$ true, then we take the path $Q_{i}=a_{2 i-2} a_{2 i-1}^{1} a_{2 i}$; if $\tau\left(u_{i}\right)=$ false, then we take the path $Q_{i}=a_{2 i-2} a_{2 i-1}^{2} a_{2 i}$. Let $D$ be the cycle formed by edge $a_{0} b_{2 m}$, all paths $Q_{i}$ for $i=1,2, \ldots, n$, and all paths $b_{2 j-1} b_{2 j-1}^{h_{j}} b_{2 j}$ for $j=1,2, \ldots, m$. Clearly $D$ is an odd cycle in $G$. For each $j$, if $z_{j}^{h_{j}}=u_{p}$ for some $p$, then $\tau\left(u_{p}\right)=$ true, and so $Q_{p}=a_{2 p-2} a_{2 p-1}^{1} a_{2 p}$. According to construction (3), $b_{2 j-1}^{h_{j}}$ is nonadjacent to $a_{2 p-1}^{1}$. Similarly, if $z_{j}^{h_{j}}=\bar{u}_{p}$, then $Q_{p}=a_{2 p-2} a_{2 p-1}^{2} a_{2 p}$, and $b_{2 j-1}^{h_{j}}$ is nonadjacent to $a_{2 p-1}^{2}$. From these two observations and the selection of $Q_{i}$, we see that $D$ contains none of the given vertex pairs.

Necessity. Suppose $D$ is an odd cycle in $G$ that contains none of the given vertex pairs. Then $D$ contains no special edges and hence is fully contained in $H$, the subgraph obtained from $G$ by deleting all special edges. Observe that $H-\left\{a_{0} b_{2 m}\right\}$ is a bipartite graph and has a plump path-like structure. From this observation and the given vertex pairs we deduce that $D$ contains edge $a_{0} b_{2 m}$ and that

(5) For each $i=1,2, \ldots, n$, either $D$ contains the path $a_{2 i-2} a_{2 i-1}^{1} a_{2 i}$ or contains the path $a_{2 i-2} a_{2 i-1}^{2} a_{2 i}$;

(6) For each $j=1,2, \ldots, m$, there exists precisely one $h_{j} \in\{1,2,3\}$ such that $D$ contains the path $b_{2 j-2} b_{2 j-1}^{h_{j}} b_{2 j}$.

Let us now define a truth assignment $\tau: U \rightarrow\{$ true, false $\}$ by setting $\tau\left(u_{i}\right)=$ true if the path specified in (5) is $a_{2 i-2} a_{2 i-1}^{1} a_{2 i}$, and $\tau\left(u_{i}\right)=$ false otherwise, for $i=1,2 \ldots, n$. It remains to show that each clause $C_{j}$ is satisfied by $\tau$. Recall the subscript $h_{j}$ in (6), if $z_{j}^{h_{j}}=u_{p}$ for some $p$, then $b_{2 j-1}^{h_{j}}$ is adjacent to $a_{2 p-1}^{2}$ in $G$, and hence $\left\{b_{2 j-1}^{h_{j}}, a_{2 p-1}^{2}\right\}$ is contained in the given vertex pairs by (3). So $D$ must contain the path $a_{2 i-2} a_{2 i-1}^{1} a_{2 i}$ by (5). According to the definition of $\tau$, we therefore have $\tau\left(u_{p}\right)=1$. It follows that $C_{j}$ is 
satisfied. Similarly, we can verify that if $z_{j}^{h_{j}}=\bar{u}_{p}$ for some $p$, then $\tau\left(u_{p}\right)=0$ and hence $C_{j}$ is also satisfied, completing the proof.

Now let us establish the main result of this paper.

Proof of Theorem 1.5. Since $A$ is an integral matrix, as shown in Schrijver [13], the three problems in our consideration are all in co- $N P$. To prove the assertion, we shall reduce the quasi-bipartite recognition problem to these problems.

For this purpose, let $G=(V, E)$ be a connected simple graph with minimum degree at least three and let $\left\{y_{1}^{1}, y_{1}^{2}\right\},\left\{y_{2}^{1}, y_{2}^{2}\right\}, \ldots,\left\{y_{t}^{1}, y_{t}^{2}\right\}$ be the given vertex pairs, where $t \geq 1$. Now add $2 t$ vertices $z_{1}^{1}, z_{1}^{2}, z_{2}^{1}, z_{2}^{2}, \ldots, z_{t}^{1}, z_{t}^{2}$ to $G$ and make both $z_{i}^{1}$ and $z_{i}^{2}$ adjacent to both $y_{i}^{1}$ and $y_{i}^{2}$ for all $i$ with $1 \leq i \leq t$. Let $G^{\prime}$ denote the resulting graph and let $A$ be the vertex-edge incidence graph of $G^{\prime}$. We claim that the following statements are equivalent:

(1) Each odd cycle in $G$ contains at least one of the given pairs;

(2) $G^{\prime}$ is a connected quasi-bipartite simple graph;

(3) The system $A \boldsymbol{x} \geq \mathbf{1}, \boldsymbol{x} \geq \mathbf{0}$ defines an integral polyhedron;

(4) The system $A \boldsymbol{x} \geq \mathbf{1}, \boldsymbol{x} \geq \mathbf{0}$ is TDI;

(5) The system $A \boldsymbol{x} \geq \mathbf{1}, \boldsymbol{x} \geq \mathbf{0}$ is box-TDI.

Indeed, since the minimum degree of $G$ is at least three, from Theorem 2.1 (with $X_{1} \cup X_{2}=\emptyset$ ) we deduce that (1) and (2) are equivalent. As $G^{\prime}$ is a connected simple graph and $G^{\prime} \neq K_{4}$, the equivalence of (2)-(5) follows instantly from Theorem 2.3 and Theorem 2.4. Thus the claim is justified.

In view of the above claim and Theorem 3.1, we conclude that the problems addressed in Theorem 1.5 are all $N P$-hard. This completes the proof of our theorem.

\section{Min-max relations}

Let $G=(V, E)$ be a graph. An edge cover of $G$ is a subset $F$ of $E$ such that each vertex of $G$ is incident to at least one edge in $F$. Clearly $G$ has an edge cover if and only if the minimum degree of $G$ is at least one. A star of $G$ consists of all the edges in $G$ incident to a vertex. Now let $\boldsymbol{w}$ be a nonnegative integral weight function defined on $E$. As defined in Schrijver [14], a $\boldsymbol{w}$-stable set of $G$ is a nonnegative integral function $\boldsymbol{y}$ defined on $V$ such that $y_{u}+y_{v} \leq w_{e}$ for each edge $e=u v$. Moreover, a $\boldsymbol{w}$-edge cover packing of $G$ is a collection $\mathcal{E}$ of edge covers (repetition is allowed) so that each edge $e$ is contained in at most $w_{e}$ members of $\mathcal{E}$. We propose to

- call $G$ König if the minimum weight of an edge cover in $G$ is equal to the maximum weight of a $w$-stable set, for any nonnegative integral $\boldsymbol{w}$; and

- call $G$ Gupta if the minimum weight of a star in $G$ is equal to the maximum size of a $\boldsymbol{w}$-edge cover packing, for any nonnegative integral $\boldsymbol{w}$. 
Graphs are so named because of the corresponding min-max theorems on bipartite graphs proved by these two authors; see Schrijver [14] for comprehensive information. The purpose of this section is to give complete characterizations of König graphs and Gupta graphs.

Theorem 4.1 A graph $G=(V, E)$ is König if and only if $G$ is quasi-bipartite, and no component of $G$ contains $K_{4}$ as a spanning subgraph.

Theorem 4.2 A graph $G=(V, E)$ is Gupta if and only if $G$ is quasi-bipartite.

Let us now proceed to the proofs of these theorems.

Proof of Theorem 4.1. Let $A$ be the vertex-edge incidence matrix of $G$. By the Edmonds-Giles theorem [8], $G$ is König if and only if the system $A \boldsymbol{x} \geq \mathbf{1}, \boldsymbol{x} \geq \mathbf{0}$ is TDI. Thus the desired statement follows instantly from Theorem 2.3.

Proof of Theorem 4.2. Without loss of generality, we may assume that $G$ is connected.

Necessity. Assume the contrary: $G$ is Gupta but not quasi-bipartite. Then $G$ contains an odd cycle $C$ such that no component of $G-V(C)$ contains only one vertex. Let $\boldsymbol{w}$ be the following weight function defined on $E: w_{e}=1$ if $e$ is an edge on $C, w_{e}=2$ if $e$ is an edge in $G-V(C)$, and $w_{e}=0$ otherwise. Then the minimum weight of a star is two. Let $\mathcal{E}$ be an arbitrary $\boldsymbol{w}$-edge cover packing in $G$. Then each edge cover in $\mathcal{E}$ contains at least $k+1$ edges from $C$, where $2 k+1$ is the length of $C$. So the size of $\mathcal{E}$ and hence the maximum size of a $\boldsymbol{w}$-edge cover packing in $G$ is at most one, which implies that $G$ is not Gupta, a contradiction.

Sufficiency. Let $\boldsymbol{w}$ be a nonnegative integral weight function defined on $E$ and let $G^{\prime}$ be the graph obtained from $G$ by replacing each edge $e$ with $w_{e}$ parallel edges. To prove that the minimum weight of a star in $G$ is equal to the maximum size of a $\boldsymbol{w}$-edge cover packing, it suffices to show that

(1) $G^{\prime}$ has a $\delta$-edge coloring such that all $\delta$ colors are represented at each vertex, where $\delta$ is the minimum degree of $G^{\prime}$.

Let us introduce some notions defined in Bondy and Murty [2] before presenting a proof of (1). Given a $k$-edge coloring $\mathcal{C}$ of $G^{\prime}$, we denote by $c(v)$ the number of distinct colors represented at $v$, and call a $k$-edge coloring $\mathcal{C}^{\prime}$ an improvement on $\mathcal{C}$ if

$$
\sum_{v \in V} c^{\prime}(v)>\sum_{v \in V} c(v)
$$

where $c^{\prime}(v)$ is the number of distinct colors represented at $v$ in the coloring $\mathcal{C}^{\prime}$. An optimal $k$-edge coloring is one which cannot be improved.

To prove (1), we consider an optimal $\delta$-edge coloring $\mathcal{C}$ of $G^{\prime}$. For convenience, let $G^{\prime \prime}$ be the subgraph obtained from $G^{\prime}$ as follows: for any vertex pair $\{i, j\}$ and any color $k$, if there is at least one edge between $i$ and $j$ with color $k$ in $G^{\prime}$ then there is precisely one edge between $i$ and $j$ in $G^{\prime \prime}$ with color $k$. Let $\mathcal{D}$ denote the restriction of $\mathcal{C}$ to $G^{\prime \prime}$. Clearly, $\mathcal{D}$ is also an optimal $\delta$-edge coloring of $G^{\prime \prime}$. Let $E_{1}, E_{2}, \ldots, E_{\delta}$ denote the color classes of $\mathcal{D}$. 
Suppose there exist a vertex $u$ in $G^{\prime \prime}$ and colors $i$ and $j$ such that $i$ is not represented at $u$ and $j$ is represented at least twice at $u$. Then, by Lemma 6.1.2 in Bondy and Murty [2], the component of $G^{\prime \prime}\left[E_{i} \cup E_{j}\right]$ that contains $u$ is an odd cycle $D$. Since $G$ is a connected quasi-bipartite graph, these exists a vertex $v$ outside $D$ such that the neighbors of $v$ in $G$ (and hence in $G^{\prime}$ ) are all contained in $D$. From the structure of $G^{\prime \prime}\left[E_{i} \cup E_{j}\right]$ we deduce that neither $i$ nor $j$ is represented at $v$. So there exists a color $k$ that is represented at least twice at $v$; let $x$ be a vertex on $D$ such that there is at least one edge $e$ between $v$ and $x$ with color $k$, and let $y$ and $z$ be the two vertices succeeding $x$ on $D$ if we traverse $D$ in cyclic order. Without loss of generality, we may assume that

(2) $u=y$; that is, both edges $x y$ and $y z$ on $D$ are colored by $j$.

Otherwise, we can obviously recolor the edges on $D$ so that $x y$ and $y z$ are both colored by $j$ and the resulting coloring of $G^{\prime}$ remains optimal. Hence (2) holds.

Let us recolor $e$ by $j$ and recolor the edge $x y$ on $D$ by $k$. By (2), the resulting $\delta$-edge coloring of $G^{\prime}$ improves $\mathcal{C}$, contradicting the optimality of $\mathcal{C}$. This completes the proof of (1) and hence the theorem.

\section{References}

[1] C. Berge, Graphs and Hypergraphs, North-Holland Publishing Company, 1976.

[2] J.A. Bondy and U.S.R. Murty, Graph Theory with Applications, Elsevier Science, 1976.

[3] M. Chudnovsky, G. Cornuéjols, X. Liu, P.D. Seymour, and K. Vušković, Recognizing Berge graphs, Combinatorica 25 (2005), 143-186.

[4] M. Chudnovsky, N. Robertson, P.D. Seymour, and R. Thomas, The strong perfect graph theorem, Ann. of Math. (2) 164 (2006), 51-229.

[5] V. Chvátal, On certain polytopes associated with graphs, J. Combin. Theory Ser. B 18 (1975), 138-154.

[6] W. Cook, L. Lovász, and A. Schrijver, A polynomial-time test for total dual integrality in fixed dimension, Math. Programming Stud. 22 (1984), 64-69.

[7] G. Cornuéjols, Combinatorial Optimization: Packing and Covering, SIAM, Philadelphia, 2001.

[8] J. Edmonds and R. Giles, A min-max relation for submodular functions on graphs, Ann. Discrete Math. 1 (1977), 185-204.

[9] J. Edmonds and R. Giles, Total dual integrality of linear inequality systems, in: Progress in Combinatorial Optimization (W.R. Pulleyblank, ed.), Academic Press, Toronto, 1984, pp. 117-129.

[10] D.R. Fulkerson, Blocking and antiblocking pairs of polyhedra, Math. Program. 1 (1971), 168-194.

[11] M. Garey and D. Johnson, Computers and Intractability: A Guide to the Theory of NP-Completeness, W.H. Freeman and Company, New York, 1979.

[12] L. Lovász, Normal hypergraphs and the perfect graph conjecture, Discrete Math. 2 (1972), 253-267.

[13] A. Schrijver, Theory of Linear and Integer Programming, John Wiley \& Sons, New York, 1986.

[14] A. Schrijver, Combinatorial Optimization - Polyhedra and Efficiency, Springer-Verlag, Berlin, 2003.

[15] P.D. Seymour, The matroids with the max-flow min-cut property, J. Combin. Theory Ser. B 23 (1977), 189-222. 